\title{
Effect of metal ions released from orthodontic mini-implants on osteoclastogenesis
}

\author{
Hataichanok Charoenpong ${ }^{1, A-F}$, Patcharee Ritprajak ${ }^{2, A, E, F}$ \\ ${ }^{1}$ Department of Orthodontics, College of Dental Medicine, Rangsit University, Thailand \\ 2 Research Unit in Integrative Immuno-Microbial Biochemistry and Bioresponsive Nanomaterials, Department of Microbiology, Faculty of Dentistry, Chulalongkorn University, \\ Bangkok, Thailand
}

A - research concept and design; B - collection and/or assembly of data; $C$ - data analysis and interpretation;

$D$ - writing the article; $E$ - critical revision of the article; $F$ - final approval of the article

\author{
Address for correspondence \\ Hataichanok Charoenpong \\ E-mail: hataichanok.@rsu.ac.th \\ Funding sources \\ The study was supported by the Research Institute \\ of Rangsit University, Thailand. \\ Conflict of interest \\ None declared

\section{Acknowledgements \\ The authors thank the Oral Biology Research Center, Faculty of Dentistry of Chulalongkorn University in Bangkok, Thailand, for facility support.}

Received on April 14, 2020

Reviewed on February 14, 2021

Accepted on March 3, 2021

Published online on August 27, 2021

Cite as

Charoenpong H, Ritprajak P. Effect of metal ions released from orthodontic mini-implants on osteoclastogenesis. Dent Med Probl. 2021;58(3):327-333. doi:10.17219/dmp/133891

DOI

10.17219/dmp/133891

Copyright

○) 2021 by Wroclaw Medical University

This is an article distributed under the terms of the

Creative Commons Attribution 3.0 Unported License (CC BY 3.0)

(https://creativecommons.org/licenses/by/3.0/).

\begin{abstract}
Background. Orthodontic mini-implants can undergo corrosion and the release of metal ions can affect cellular behavior. Osteoclasts are involved in orthodontic tooth movement and implant stability. Osteoclasts and their precursors can be exposed to metal ions released from orthodontic mini-implants.

Objectives. This study aimed to investigate the effect of metal ions released from orthodontic miniimplants on human osteoclastogenesis.

Material and methods. Stainless steel and titanium alloy mini-implants were separately immersed in culture media for 14 days (days 1-14), and then moved to new media for a further 14 days (days 15-28). The concentration of the released metal ions was measured. Osteoclast precursors derived from human CD14+ monocytes were cultured in these media and in a control medium without mini-implant immersion. Cell viability, the number of osteoclasts and the area of resorption were investigated.

Results. A higher concentration of metal ions was detected during the first 14 days as compared to the control. The concentration of these metal ions then declined after this period. The viability of osteoclast precursors was not affected by the released metal ions. There was a significant reduction in the number of osteoclasts when cultured in the medium with the titanium alloy mini-implants immersed for days 1-14. The area of resorption was also significantly reduced in this group. The media with the titanium alloy mini-implants immersed for days 15-28 and with the stainless steel mini-implants immersed for both study periods did not show statistically significant changes in the number of osteoclasts.

Conclusions. Metal ions were released from orthodontic mini-implants in the early period and declined thereafter. Metal ions released from titanium mini-implants in the early period inhibited osteoclastogenesis, while metal ions from stainless steel mini-implants had no effect on osteoclast differentiation.
\end{abstract}

Keywords: cytotoxicity, biocompatibility, osteoclast, metal ion, orthodontic mini-implant 


\section{Introduction}

Orthodontic mini-implants, also known as orthodontic miniscrews or temporary anchorage devices, have increased in popularity for use in orthodontic treatment in recent decades. Mini-implants are placed into bone during orthodontic treatment to act as skeletal anchorages in order to provide tooth movement, which is impossible with the use of tooth-borne anchorages. ${ }^{1}$

Materials commonly used for orthodontic miniimplants are titanium or titanium alloy and stainless steel. ${ }^{2}$ All metals that are in contact with a biological fluid can undergo corrosion and metal ions are released into the biological system. ${ }^{3-5}$ Orthodontic mini-implants undergo corrosion. Metal ions released from orthodontic miniimplants can be detected locally in the saliva of patients after mini-implant placement, and also systemically in remote organs in animal studies. ${ }^{6,7}$

Metal ions can affect various cell types in the human body, including osteoclasts. ${ }^{8,9}$ When mini-implants are placed into bone, osteoclasts and their precursors can be exposed to the released metal ions. Osteoclasts are bone-resorbing cells involved in implant stability; thus, any disturbance of osteoclast activity can be related to implant failure. ${ }^{10,11}$ Osteoclasts are crucial in orthodontic tooth movement and the inhibition of osteoclasts may compromise tooth movement. In certain situations, the tooth is expected to move toward the mini-implant during the treatment period, such as in retromolar placement for distalization. ${ }^{12}$ Prolonged effects of metal ions released from mini-implants on osteoclastogenesis may affect tooth movement in such cases. In a previous report, mini-implants were used to create micropores in bone to accelerate tooth movement. ${ }^{13}$ In such circumstances, the teeth are quickly moved toward the sites of the previously placed mini-implants. It is important to recognize and better understand factors related to mini-implant stability as well as to determine ways of utilizing mini-implants for different purposes. Therefore, this study aimed to investigate the effect of metal ions released from orthodontic mini-implants on osteoclastogenesis during different periods of time.

\section{Material and methods}

\section{Preparing culture media with mini-implant immersion}

Titanium alloy $\left(\mathrm{Ti}_{6} \mathrm{Al}_{4} \mathrm{~V}\right)$ mini-implants (Renew Biocare Corporation Asia Pacific, Taipei, Taiwan) and stainless steel mini-implants (Bio-Ray Biotech Instrument Co., Ltd., Taipei, Taiwan) were used in this study. The titanium alloy consisted of titanium-based metal with $6 \%$ aluminum, $4 \%$ vanadium and trace amounts of other elements.
The stainless steel mini-implants contained $63 \%$ iron, $17 \%$ chromium, $14 \%$ nickel, $3 \%$ molybdenum, $2 \%$ manganese, and $1 \%$ other elements.

The titanium alloy and stainless steel mini-implants were separately immersed in culture media containing $\alpha-M E M$ (Minimum Essential Medium) (HyClone, Logan, USA) at a ratio of $1 \mathrm{~mL} / 0.1 \mathrm{~g}$ and $1 \mathrm{~mL} / 0.2 \mathrm{~g}$, respectively. These ratios are recommended by the International Standards Organization (ISO) and the Japanese Ministry of Health, Labour and Welfare. Both kinds of mini-implants were immersed in the media for 14 days (days 1-14), and then moved to new media for another 14 days (days 15-28). The media were kept in a humidified atmosphere at $37^{\circ} \mathrm{C}$ during the immersion periods. The media from each period were then collected and divided into 2 parts. One was used to measure the concentration of metal ions, and the other was used to culture osteoclasts in order to determine their number and cell viability, and the area of resorption.

\section{Measurement of metal ion release}

The concentration of metal ions was detected using inductively coupled plasma mass spectrometry (ICP-MS) (iCAPTM RQ ICP-MS; Thermo Fisher Scientific, Bremen, Germany). The instrument parameters are shown in Table 1. The concentrations of iron, chromium and nickel ions - the 3 main components of stainless steel miniimplants - were measured in the media in which the stainless steel mini-implants were immersed. The concentrations of titanium, aluminum and vanadium ions - the components of titanium alloy mini-implants - were measured in the media in which the titanium alloy mini-implants were immersed.

\section{Osteoclast differentiation}

Human osteoclast precursors were derived from monocytes obtained from the peripheral blood of healthy donors. The protocol was approved by the Ethics Committee of the Research Institute of Rangsit University, Thailand (RSEC 33/2559) and informed consent was obtained from the subjects. Briefly, $50 \mathrm{~mL}$ of peripheral venous blood was collected from each healthy donor. Peripheral blood mononuclear cells (PBMCs) were separated through density gradient centrifugation, using Histopaque ${ }^{\circledR}-1077$ (Sigma-Aldrich, St. Louis, USA). CD $14^{+}$monocytes were further sorted

Table 1. General operational conditions of inductively coupled plasma mass spectrometry (ICP-MS)

\begin{tabular}{|lc|}
\hline \multicolumn{1}{|c|}{ Parameter } & Value \\
\hline Plasma power & $1,550 \mathrm{~W}$ \\
Cool flow & $14 \mathrm{~L} / \mathrm{min}$ \\
Auxiliary flow & $0.8 \mathrm{~L} / \mathrm{min}$ \\
Nebulizer flow & $1.043 \mathrm{~L} / \mathrm{min}$ \\
Dwell time & $0.1 \mathrm{~s}$ \\
\hline
\end{tabular}


using human CD14 MicroBeads (Miltenyi Biotec, Auburn, USA). The cells were then plated on a 96-well plate at a density of $10^{5}$ cells per well to determine cell viability and the number of osteoclasts. For the detection of a resorption pit, $7 \times 10^{4}$ cells were plated in each well of a 96-well plate containing dentin slices, $5 \mathrm{~mm}$ in diameter, which were prepared from cylindrical elephant ivory cut to a thickness of $300 \mu \mathrm{m}$, as previously described. ${ }^{14}$ The cells in each well were maintained in $50 \mu \mathrm{L}$ of the culture media in which the mini-implants were previously immersed or in a medium without mini-implant immersion, which served as the control. All of the culture media were supplemented with $10 \%$ fetal bovine serum (FBS) (Gibco $^{\mathrm{TM}}$, Thermo Fisher Scientific, Rochester, USA), 0.2 mM GlutaMAX ${ }^{\mathrm{TM}}$ (Gibco), $100 \mathrm{U} / \mathrm{mL}$ penicillin (Gibco), $100 \mathrm{mg} / \mathrm{mL}$ streptomycin (Gibco), $0.25 \mu \mathrm{g} / \mathrm{mL}$ amphotericin B (Gibco), $25 \mathrm{ng} / \mathrm{mL}$ recombinant human receptor activator of nuclear factor kappa-B ligand (rh-RANKL) (PeproTech Inc., Rocky Hill, USA), and $25 \mathrm{ng} / \mathrm{mL}$ recombinant human macrophagecolony stimulating factor (rhM-CSF) (PeproTech Inc.). The media were changed every 2-4 days. At least 3 different lines of osteoclast precursors were used and at least 2 independent experiments were performed.

\section{Cell viability assay}

The viability of osteoclast precursors was determined after $20 \mathrm{~h}$ of cell culture. Briefly, the cells were incubated with 3-(4,5-dimethylthiazol-2-yl)-2,5-diphenyltetrazolium bromide (MTT) solution (USB Corporation, Cleveland, USA) for $40 \mathrm{~min}$ at $37^{\circ} \mathrm{C}$ with $5 \% \mathrm{CO}_{2}$. Formazan crystals were dissolved with a solubilization agent containing 1:9 dimethyl sulfoxide (DMSO) (Merck, Darmstadt, Germany) and glycine buffer (0.1 M glycine/0.1 M sodium chloride; $\mathrm{pH} 10)$. The absorbance of the solution at $570 \mathrm{~nm}$ was then measured using a microplate reader $\left(\mathrm{EL} \times 800^{\mathrm{TM}}\right.$; BioTek Instruments, Winooski, USA).

\section{Determination of the number of osteoclasts}

After 14 days of differentiation, the cells were stained for tartrate-resistant acid phosphatase (TRAP) and the number of osteoclasts in each sample was determined. Briefly, the cells were fixed with $10 \%$ neutral buffered formalin followed by washing with $95 \%$ ethanol. The cells were then stained for TRAP with $0.1 \mathrm{mg} / \mathrm{mL}$ naphthol AS-MX phosphate (Sigma-Aldrich) and $0.5 \mathrm{mg} / \mathrm{mL}$ fast red violet LB salt (Sigma-Aldrich) in $50 \mathrm{mM}$ sodium acetate buffer (pH 5.0) containing $50 \mathrm{mM}$ sodium tartrate for $20 \mathrm{~min}$. After washing, the cells were visualized under bright-field microscopy. Images were obtained from at least 3 fields for each culture well with the use of $\times 100$ stage objectives (Olympus DP72; Olympus, Tokyo, Japan). Cells that were TRAP-positive and contained at least 3 nuclei were counted as osteoclasts.

\section{Determination of resorptive activity}

After 14 days of osteoclast differentiation, the cells were removed from the dentin slices by sonication in $25-30 \%$ ammonium hydroxide. After washing, the dentin slices were stained with $1 \mathrm{mg} / \mathrm{mL}$ toluidine blue and visualized under bright-field microscopy. Images were obtained from at least 3 fields for each dentin slice under $\times 100$ stage objectives (Olympus DP72; Olympus). The area of resorption was measured using the measurement tool in the cellSens software (Olympus).

\section{Statistical analysis}

Themean concentration of metal ions in the media in which the mini-implants were immersed was compared to the control using the one-sample $t$ test. Cell viability was analyzed by comparing the mean absorbance value among all groups using the one-way analysis of variance (ANOVA). The number of osteoclasts was calculated as fold changes compared to the control from the same precursor lines. The mean fold changes in the number of osteoclasts were compared among all groups using the one-way ANOVA. The least significant difference (LSD) post-hoc test was performed for any significant difference identified with ANOVA. The resorption area was calculated as a fold change compared to the control from the same precursor lines. The mean fold change in the resorption area in the test group was compared to that of the control using Student's $t$ test.

\section{Results}

\section{Metal ions released from orthodontic mini-implants}

The presence of metal ions in the media in which the mini-implants of either material were immersed was confirmed. The concentrations of iron, chromium and nickel ions were significantly higher in the medium with the stainless steel mini-implants immersed for days $1-14$ as compared to the control $(p<0.01, p<0.05$ and $p<0.01$, respectively) (Table 2 ). The medium with the titanium alloy mini-implants immersed for days 1-14 had significantly higher concentrations of titanium and vanadium ions as compared to the control $(p<0.01)$ (Table 2). This indicates a substantial release of metal ions from the mini-implants of either material during this period. At days 15-28, the level of metal ions declined until no significant difference was observed for all metal ions except titanium ions; their concentration was significantly higher than in the control medium $(p<0.01)$. We were unable to detect any increase in the concentration of aluminum ions after mini-implant immersion in either period due to a high background amount of aluminum ions in the media. Taken together, 
Table 2. Concentration of metal ions in the control medium without mini-implant immersion and the media in which the stainless steel and titanium alloy mini-implants were immersed $[\mu \mathrm{g} / \mathrm{L}, \mathrm{ppb}]$

\begin{tabular}{|c|c|c|c|c|c|c|c|}
\hline Group & $\mathrm{Fe}$ & $\mathrm{Cr}$ & $\mathrm{Ni}$ & Group & $\mathrm{Ti}$ & V & $\mathrm{Al}$ \\
\hline control & $<0.1$ & $<0.02$ & 3.50 & control & 1.50 & $<0.02$ & 138.50 \\
\hline SS 1-14 & $9.67^{* *}$ & $5.67^{*}$ & $8.83^{* *}$ & Ti 1-14 & $23.17^{* *}$ & $3.83^{* *}$ & 134.33 \\
\hline SS $15-28$ & $<0.1$ & $<0.02$ & 2.67 & Ti 15-28 & $15.33^{* *}$ & 0.33 & 117.50 \\
\hline
\end{tabular}

SS - stainless steel; Ti - titanium alloy; 1-14 - days 1-14 of immersion; 15-28-days 15-28 of immersion;

${ }^{*} p<0.05$ compared to the control; ${ }^{* *} p<0.01$ compared to the control.

the results confirm that metal ions were released from the orthodontic mini-implants of either material. The release of metal ions was higher during the initial period and decreased over time, with a prolonged release of titanium ions from the titanium alloy mini-implants.

\section{Viability of osteoclast precursors}

The number of viable osteoclast precursors indicated by the absorbance measured after the MTT assay was not statistically different while comparing the control medium, the media in which the titanium alloy miniimplants were immersed and the media in which the stainless steel mini-implants were immersed $(p \geq 0.05)$ (Fig. 1). This indicates that the presence of metal ions from both the stainless steel and titanium alloy mini-implants used in this study was not cytotoxic to osteoclast precursors. Thus, metal ions influenced the ability of osteoclasts to differentiate rather than caused cell death, which is confirmed by the results presented below, regarding the number and resorption ability of osteoclasts.

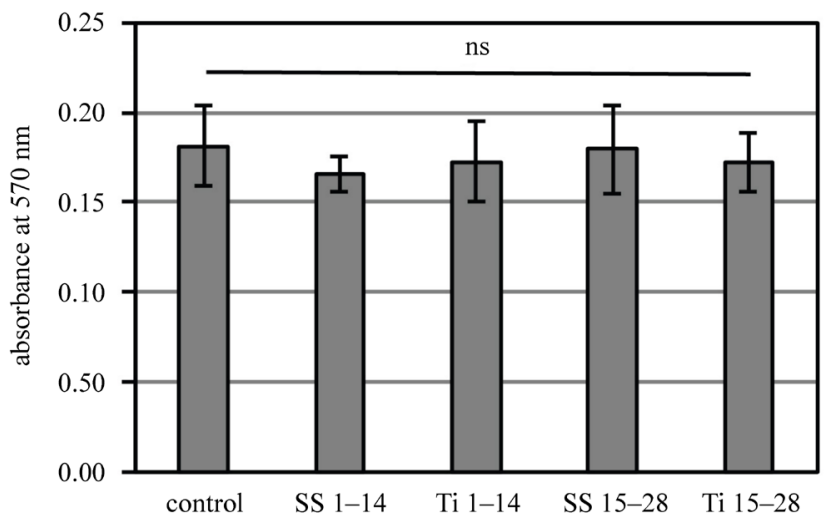

Fig. 1. Viability of osteoclast precursors. The absorbance measured after the MTT assay indicated no statistically significant differences in the number of viable cells between all media groups

ns - not significant ( $p \geq 0.05)$.

Data presented as mean \pm standard deviation $(M \pm S D)(n=3)$.

\section{Effect on osteoclastogenesis}

There was a significant reduction in the number of osteoclasts when cultured in the medium with the titanium alloy mini-implants immersed for days $1-14(p<0.05)$.
For the medium with the stainless steel mini-implants immersed for days 1-14, although there was a tendency toward a reduction in the numbers of osteoclasts, no significant change was observed $(p \geq 0.05)$ (Fig. 2).

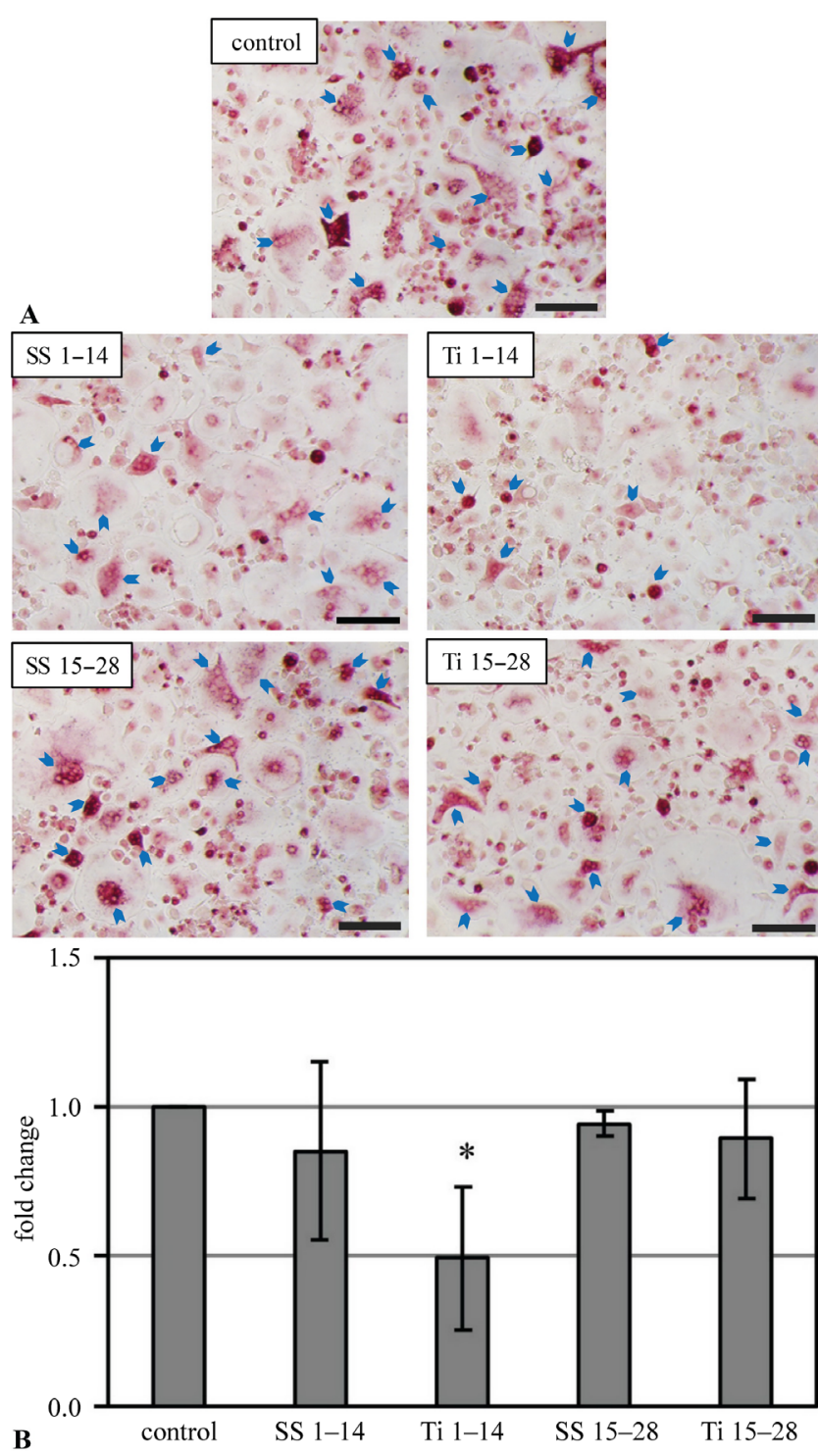

Fig. 2. Changes in the number of osteoclasts. A. Representative images of the TRAP staining of osteoclasts in the control and mini-implant immersion media after 14 and 28 days of differentiation. B. The number of osteoclasts in the mini-implant immersion media was calculated as fold changes compared to the control. Fold changes in the osteoclast number compared to the control in SS 1-14, Ti 1-14, SS 15-28, and Ti 15-28 were $0.85 \pm 0.30,0.49 \pm 0.24,0.94 \pm 0.05$, and $0.89 \pm 0.20$, respectively

* statistically significant $(p<0.05)$. Data presented as $M \pm S D(n=4)$. 
For days 15-28, no significant change in the osteoclast number was noted for the mini-implants of either material $(p \geq 0.05)$ (Fig. 2).

The ability of osteoclasts to resorb the mineralized tissue was further researched in the medium with the titanium alloy mini-implants immersed for days $1-14$; in this group there was a significant decrease in the osteoclast number. It was found that the area of resorption was also significantly reduced in this case $(p<0.05)$ (Fig. 3). Collectively, the results indicate that there was a significant suppression of osteoclastogenesis in the presence of metal ions released from the titanium alloy mini-implants at days $1-14$.

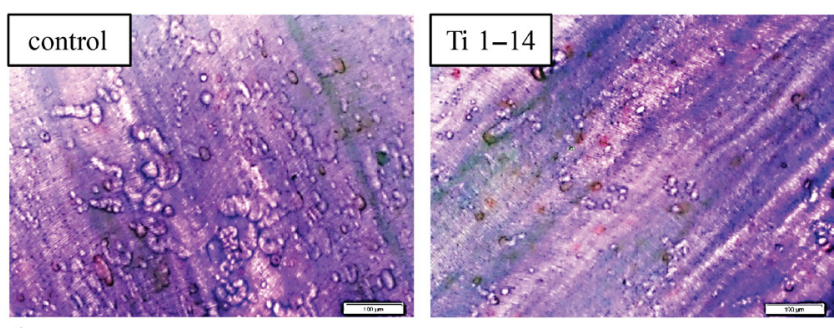

A

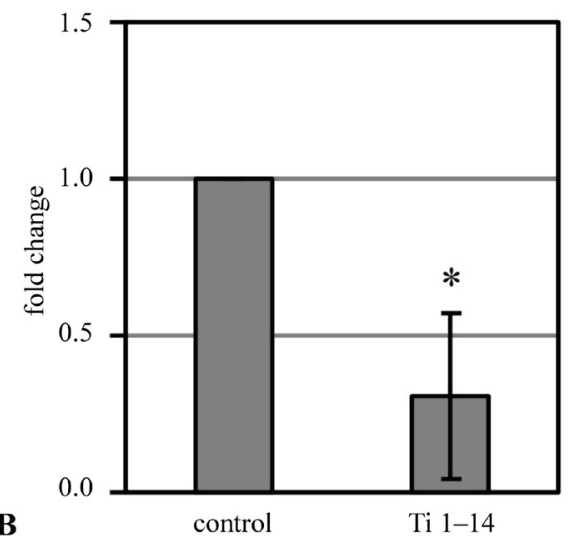

Fig. 3. Changes in the area of resorption. A. Representative images of resorption pits on the dentin slices in the control medium compared to the medium with the titanium alloy mini-implants immersed for days $1-14$. B. The area of resorption of the osteoclasts cultured in the medium with the titanium alloy mini-implants immersed for days 1-14 was calculated as a fold change compared to the control. A fold change in the area of resorption in the Ti 1-14 group compared to the control was $0.31 \pm 0.26$

* statistically significant $(p<0.05)$

Data presented as $M \pm S D(n=3)$.

\section{Discussion}

The results of this study confirm that metal ions were released from both stainless steel and titanium alloy orthodontic mini-implants. After the initial release, it is possible that soluble metal ions transformed into solid corrosion precipitates, which may have lowered the concentrations of metal ions detected in the solution over time. However, a previous study indicated that within a 28-day period, the level of metal ions released from titanium alloy mini-implants continued to increase with longer immersion durations from 1 day to 28 days. $^{15}$
In this study, the release of metal ions from orthodontic mini-implants was found to be higher when the miniimplants were initially in contact with the media and decreased over time. This could be due to the formation of a thin barrier film on the surface of the initially corroded mini-implants, which served to prevent further corrosion of deeper metal atoms. ${ }^{16}$ Nonetheless, since mechanical force may disrupt this barrier layer, ${ }^{16}$ it should be noted that when force is applied to mini-implants in the patient's mouth, the release of metal ions may continue beyond the periods investigated in this study.

The stainless steel mini-implants used in this study were made of type 316L stainless steel from the American Iron and Steel Institute. This material is recommended by the American Society for Testing and Materials (ASTM International) for use as implant material. Stainless steel 316L contains molybdenum and has a low carbon content, which minimizes intergranular corrosion, and also makes the material more resistant to chlorine-bearing solutions. ${ }^{2}$

The presence of metal ions at high concentrations can be cytotoxic to cells, ${ }^{17-19}$ but with biocompatible material, the release of metal ions should not affect cell viability. This study demonstrated that the concentrations of metal ions released from the orthodontic mini-implants of either material were within a non-cytotoxic range for osteoclast precursor cells. This result is consistent with previous studies which investigated the biocompatibility and cytotoxicity of orthodontic miniscrews in L929, HaCaT, HGF, and U2OS cells. ${ }^{17,20}$ They reported that there was no cytotoxicity due to titanium mini-implants when in contact with a physiologic $\mathrm{pH}$ solution. ${ }^{17,20}$

Different metal ions may have different effects on osteoclasts. However, this study was not designed to separately evaluate the effect of each type of metal ions. Instead, we employed the direct elution of orthodontic mini-implants, so the effect on osteoclasts was from the combination of various metal ions released in a proportion that corresponded to the corrosion rate of each ion type. This should represent the release of metal ions in clinical situations, in which mini-implants are inserted into the patient's bone.

The results of the present study indicate that metal ions released from the titanium alloy mini-implants during the initial period (days 1-14) had a suppressive effect on osteoclastogenesis. These results support the findings of previous studies, which found that titanium ions might reduce the number of osteoclasts and resorption pits. ${ }^{21,22}$ Vanadium ions were shown to have an inhibitory effect on osteoclasts in previous studies. ${ }^{18,22}$ Metal ions released from the titanium alloy mini-implants during the later period (days 15-28) did not affect the number of osteoclasts. This could be due to the fact that the concentrations of metal ions found in this period were too low to have an effect on osteoclasts.

The effect of iron and chromium ions - the components of stainless steel - on osteoclasts has been reported previously with conflicting results. Iron ions were 
reported to stimulate osteoclast formation by Jia et al., ${ }^{23}$ yet significantly reduced the osteoclast number in a study by Rousselle et al. ${ }^{22}$ Chromium was found to have no effect on osteoclasts when using animal cells, ${ }^{21}$ whereas a study using human PBMCs found an inhibitory effect of $\mathrm{Cr}^{6+}$ and a biphasic effect of $\mathrm{Cr}^{3+}$ on osteoclasts. ${ }^{24} \mathrm{In}$ the present study, metal ions released from the stainless steel mini-implants in both study periods were found not to affect the number of osteoclasts. This could result from each of these metal ions having no effect on osteoclasts or the effect on osteoclasts was neutralized, since some of these metal ions may have an inhibitory effect, while others have a stimulatory effect. Additionally, since the concentrations of the ions of each metal producing the effect on particular cells is varied, it is also possible that the level of metal ions released from the stainless steel miniimplants did not reach a sufficient concentration to affect osteoclasts.

The suppression of osteoclastogenesis by metal ions from the titanium alloy mini-implants did not result from the death of monocyte precursors according to cell viability results (Fig. 1). Therefore, a reduction in the osteoclast number must have been a result of disturbance in the differentiation and maturation process of osteoclasts, which is regulated by various mechanisms. Zinc has been reported to suppress osteoclasts by inhibiting the $\mathrm{Ca}^{2+}$-calcineurin-NFATc1 signaling pathway. ${ }^{25}$ Meanwhile, magnesium has been shown to inhibit osteoclasts through the inhibition of the nuclear factor kappa $B$ (NF-kB) pathway. ${ }^{26}$ However, the mechanisms underlying the inhibition of osteoclasts by metal ions from titanium mini-implants require further investigation.

Changes in osteoclastic activity may affect miniimplant stability. It has been reported that the local delivery of bisphosphonate - a drug that inhibits osteoclastic activity - can enhance mini-implant stability. ${ }^{27}$ Therefore, the results of the present study suggest that metal ions released from the titanium alloy mini-implants during the early period may be one factor that promotes the stability of orthodontic mini-implants according to their inhibiting effect on osteoclastogenesis.

Early mini-implant loading is beneficial in terms of reducing treatment time; however, stability is of concern in instances of early loading. Previous studies reported that the early loading of orthodontic force onto mini-implants did not compromise their stability as compared to delayed loading. ${ }^{28,29}$ The results of this study also support the early loading of the titanium alloy mini-implants, since a decrease in osteoclastic activity should minimize the likelihood of the loosening of mini-implants during the first 2 weeks.

Recently, orthodontic mini-implants have been reported to be used for micro-osteoperforation to accelerate tooth movement. ${ }^{13,30}$ In this procedure, mini-implants are placed and removed at the alveolar bone adjacent to the teeth to create microperforations prior to rapid tooth movement toward the mini-implant sites. Since the stimulation of osteoclasts is expected at mini-implant placement sites following this procedure, stainless steel miniimplants would be preferred according to the results of this study.

After 2 weeks, the release of metal ions from orthodontic mini-implants decreased until no effect on osteoclasts was observed for the mini-implants made of either material. According to this result, in certain cases in which the teeth must be moved toward mini-implants during treatment, such as in retromolar placement for molar distalization, after a 2-week period, metal ions from the mini-implant should no longer affect osteoclasts and tooth movement.

\section{Conclusions}

This study demonstrated that metal ions were released from stainless steel and titanium alloy orthodontic miniimplants at non-cytotoxic levels for osteoclast precursor cells. The release of these metal ions was found to be higher during the initial period, but then declined over time. Finally, metal ions released from titanium mini-implants during the initial period inhibited osteoclastogenesis, while metal ions from stainless steel mini-implants were found not to affect osteoclast differentiation.

\section{ORCID iDs}

Hataichanok Charoenpong (1) https://orcid.org/0000-0002-2504-7740 Patcharee Ritprajak (1) https://orcid.org/0000-0003-1392-4061

\section{References}

1. Leung MTC, Lee TCK, Rabie ABM, Wong RWT. Use of miniscrews and miniplates in orthodontics. J Oral Maxillofac Surg. 2008;66(7):1461-1466. doi:10.1016/j.joms.2007.12.029

2. Sana S, Manjunath G. Mini-implant materials: An overview. IOSR J Dent Med Sci. 2013;7(2):15-20. doi:10.9790/0853-0721520

3. Dimić I, Cvijović-Alagić I, Rakin M, Bugarski B. Analysis of metal ion release from biomedical implants. Metall MaterEng. 2013;19(2):167-176.

4. Eliaz N. Corrosion of metallic biomaterials: A review. Materials (Basel). 2019;12(3):407. doi:10.3390/ma12030407

5. Hanawa T. Metal ion release from metal implants. Mater Sci Eng C. 2004;24(6-8):745-752. doi:10.1016/j.msec.2004.08.018

6. Blaya MG, Blaya DS, Mello P, Flores EMM, Hirakata LM. Titanium alloy miniscrews for orthodontic anchorage: An in vivo study of metal ion release. Rev Odonto Cienc. 2011;26(3):209-214. doi:10.1590/S1980-65232011000300003

7. Sigueira de Morais L, Serra GG, Albuquerque Palermo EF, et al. Systemic levels of metallic ions released from orthodontic miniimplants. Am J Orthod Dentofacial Orthop. 2009;135(4):522-529. doi:10.1016/j.ajodo.2007.04.045

8. Noronha Oliveira M, Schunemann WVH, Mathew MT, et al. Can degradation products released from dental implants affect periimplant tissues? J Periodontal Res. 2018;53(1):1-11. doi:10.1111/jre.12479

9. Sansone V, Pagani D, Melato $M$. The effects on bone cells of metal ions released from orthopaedic implants. A review. Clin Cases Miner Bone Metab. 2013;10(1):34-40. doi:10.11138/ccmbm/2013.10.1.034

10. Jiang Y, Jia T, Wooley PH, Yang SY. Current research in the pathogenesis of aseptic implant loosening associated with particulate wear debris. Acta Orthop Belg. 2013;79(1):1-9.

11. Minkin C, Marinho VC. Role of the osteoclast at the bone-implant interface. Adv Dent Res. 1999;13:49-56. doi:10.1177/08959374990130011401 
12. Poletti L, Silvera AA, Huanca Ghislanzoni LT. Dentoalveolar class III treatment using retromolar miniscrew anchorage. Prog Orthod. 2013;14:7. doi:10.1186/2196-1042-14-7

13. Cheung T, Park J, Lee $D$, et al. Ability of mini-implant-facilitated micro-osteoperforations to accelerate tooth movement in rats. Am J Orthod Dentofacial Orthop. 2016;150(6):958-967. doi:10.1016/j.ajodo.2016.04.030

14. Rumpler $M$, Würger $T$, Roschger $P$, et al. Microcracks and osteoclast resorption activity in vitro. Calcif Tissue Int. 2012;90(3):230-238. doi:10.1007/s00223-011-9568-z

15. El Sawy AA, Shaarawy MA. Evaluation of metal ion release from $\mathrm{Ti}_{6} \mathrm{Al}_{4} \mathrm{~V}$ and $\mathrm{Co}-\mathrm{Cr}$-Mo casting alloys: In vivo and in vitro study. J Prosthodont. 2014;23(2):89-97. doi:10.1111/jopr.12067

16. Patterson SP, Daffner RH, Gallo RA. Electrochemical corrosion of metal implants. AJR Am J Roentgenol. 2005;184(4):1219-1222. doi:10.2214/ajr.184.4.01841219

17. Galeotti A, Uomo R, Spagnuolo $\mathrm{G}$, et al. Effect of $\mathrm{pH}$ on in vitro biocompatibility of orthodontic miniscrew implants. Prog Orthod. 2013;14:15. doi:10.1186/2196-1042-14-15

18. König MA, Gautschi OP, Simmen HP, Filgueira L, Cadosch D. Influence of vanadium ${ }^{4+}$ and ${ }^{5+}$ ions on the differentiation and activation of human osteoclasts. Int J Biomater. 2017;2017:9439036. doi:10.1155/2017/9439036

19. Puleo DA, Huh WW. Acute toxicity of metal ions in cultures of osteogenic cells derived from bone marrow stromal cells. J Appl Biomater. 1995;6(2):109-116. doi:10.1002/jab.770060205

20. Costa Alves CB, Segurado MN, Leandro Dorta MC, Dias FR, Lenza MG, Lenza MA. Evaluation of cytotoxicity and corrosion resistance of orthodontic mini-implants. Dental Press J Orthod. 2016;21(5):39-46. doi:10.1590/2177-6709.21.5.039-046.oar

21. Nichols KG, Puleo DA. Effect of metal ions on the formation and function of osteoclastic cells in vitro. J BiomedMater Res. 1997;35(2):265-271. doi:10.1002/(sici)1097-4636(199705)35:2<265::aid-jbm14>3.0.co;2-g

22. Rousselle AV, Heymann D, Demais V, Charrier C, Passuti N, Baslé MF. Influence of metal ion solutions on rabbit osteoclast activities in vitro. Histol Histopathol. 2002;17(4):1025-1032. doi:10.14670/HH-17.1025

23. Jia $P, X u$ YJ, Zhang ZL, et al. Ferric ion could facilitate osteoclast differentiation and bone resorption through the production of reactive oxygen species. J Orthop Res. 2012;30(11):1843-1852. doi:10.1002/jor.22133

24. Andrews RE, Shah KM, Wilkinson JM, Gartland A. Effects of cobalt and chromium ions at clinically equivalent concentrations after metal-on-metal hip replacement on human osteoblasts and osteoclasts: Implications for skeletal health. Bone. 2011;49(4):717-723. doi:10.1016/j.bone.2011.06.007

25. Park KH, Park B, Yoon DS, et al. Zinc inhibits osteoclast differentiation by suppression of $\mathrm{Ca}^{2+}$-Calcineurin-NFATc1 signaling pathway. Cell Commun Signal. 2013;11:74. doi:10.1186/1478-811X-11-74

26. Zhai Z, Qu X, Li H, et al. The effect of metallic magnesium degradation products on osteoclast-induced osteolysis and attenuation of NF-KB and NFATC1 signaling. Biomaterials. 2014;35(24):6299-6310. doi:10.1016/j.biomaterials.2014.04.044

27. Cuairán C, Campbell PM, Kontogiorgos E, Taylor RW, Melo AC Buschang $\mathrm{PH}$. Local application of zoledronate enhances miniscrew implant stability in dogs. Am J Orthod Dentofacial Orthop. 2014;145(6):737-749. doi:10.1016/j.ajodo.2014.01.020

28. Kuroda S, Sugawara Y, Deguchi T, Kyung HM, Takano-Yamamoto T. Clinical use of miniscrew implants as orthodontic anchorage: Success rates and postoperative discomfort. Am J Orthod Dentofacial Orthop. 2007;131(1):9-15. doi:10.1016/j.ajodo.2005.02.032

29. Migliorati M, Drago S, Gallo F, et al. Immediate versus delayed loading: Comparison of primary stability loss after miniscrew placement in orthodontic patients - a single-centre blinded randomized clinical trial. Eur J Orthod. 2016;38(6):652-659. doi:10.1093/ejo/cjv095

30. Kim J, Kook YA, Bayome $M$, et al. Comparison of tooth movement and biological response in corticotomy and micro-osteoperforation in rabbits. Korean J Orthod. 2019;49(4):205-213. doi:10.4041/kjod.2019.49.4.205 